\title{
Persistent visual disturbances after concussion
}

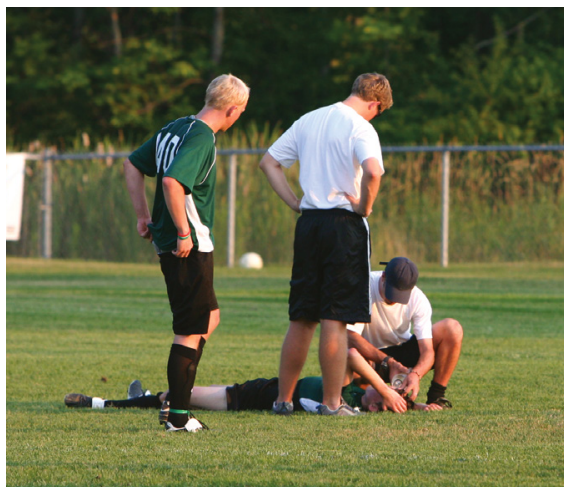

CPD

Premkumar Gunasekaran,

Christopher Hodge, Kathryn Rose, Clare L Fraser

\section{Background}

Ocular dysfunction, including eye movement defects, has been documented in up to $69 \%$ of patients with concussion. However, standard sports-related concussion assessment protocols do not typically include any clinical examination of the ocular system.

\section{Objective}

The aim of this article is to inform general practitioners (GPs) about ocular defects associated with concussion, identify test procedures and highlight the important role of GPs within the concussion paradigm.

\section{Discussion}

Ocular dysfunction that commonly occurs with concussion includes abnormalities of accommodation, convergence, saccades and smooth pursuits. This may cause blurred vision, double vision, ocular pain and difficulty with close work. Symptoms can severely affect daily work, school or play activities. Patients complaining of extended ocular symptoms following concussion should be referred to an ophthalmologist for a complete ocular assessment.
WITH AN INCREASED emphasis on awareness and diagnosis of concussion in both professional and amateur sports, the frequency of reported concussions has significantly increased over the past decade. Australian data suggest that the frequency of reported concussions increased by $60.5 \%$ between 2002 and 2011 , leading to an annual incidence of $>4500$ hospitalisations from sporting events. ${ }^{1,2}$ The costs associated with hospitalisation from these incidents was approximately $\$ 18$ million, representing a significant burden on the health system. ${ }^{2}$ The majority of traumatic brain injuries occur in patients aged 15-64 years during participation in sports. ${ }^{1}$ Although most cases resolve spontaneously without medical intervention over a period of 7-10 days, a subset of patients will report ongoing symptoms. ${ }^{3}$ Optimal diagnosis and, where indicated, treatment is therefore essential to reduce the impact on either school, work or daily activities and subsequent quality of life. ${ }^{4}$

Concussion is a diffuse brain injury. As such, signs and symptoms on presentation may be both complex and highly variable. Immediately following impact, the patient may have confusion, dizziness and amnesia. Additional early symptoms include headaches, difficulty concentrating on tasks, sensitivity to light and visual disturbances. ${ }^{5}$ Despite previous misconceptions, concussion often does not involve the loss of consciousness, which is reported in $<10 \%$ of cases. $^{6}$

The widely endorsed Sports Concussion Assessment Tool 5th Edition (SCAT5) and Concussion Recognition Tool (CRT) have shown high sensitivity and specificity in detecting concussion at the sports arena. However, the use of these tests is often restricted to professional or semi-professional sporting events, where trained medical professionals are more readily available. ${ }^{78}$ Accordingly, it has been reported that up to $90 \%$ of players may not be aware of a potential concussion diagnosis and go unassessed by health professionals. ${ }^{9}$ This presents particular difficulties for general practitioners (GPs), who will often see patients at a time after the concussive event. Differentiation of non-specific concussion symptoms from pre-existing conditions is a further confounder.

As more than half of the brain's pathways are dedicated to vision and eye movement control, a diffuse brain injury such as concussion will often affect the visual system. ${ }^{10}$ Research has found that visual disorders may occur in up to $69 \%$ of patients following mild traumatic brain injuries. ${ }^{11}$ For children, adolescents and young adults, this may have a detrimental 
effect on their ability to complete academic activities. In adults, this may lead to difficulties performing crucial daily activities such as driving and coping with work. Early impact or sideline tests include the question, 'Have you noticed blurred or double vision?'; however, the potential impact on the visual system following concussion may require more in-depth clinical examination and investigation. This literature review aims to inform GPs about the ocular defects associated with concussion and to highlight the GPs' important role within the concussion paradigm.

\section{Methods}

A PubMed search using Medical Subject Headings (MeSH) terms including 'brain concussion', 'concussion', 'vision', 'eye movement' or 'visual dysfunction' identified 495 papers. After screening for duplicates, this was reduced to 220 papers. These were screened individually; only peer-reviewed, human-based studies published within the past 10 years were considered. Of these papers, only those that tested oculomotor dysfunction following a concussion were included in this analysis (15 papers). This search was conducted on 10 May 2019.

\section{Oculomotor dysfunction}

Studies in both adolescent and adult patients have described persistent clinical features affecting the visual system following a concussion (Table 1). These may include diplopia (double vision), decreased visual attention, abnormal pupils and an increase in abnormal eye tracking movements. ${ }^{12}$ However, the literature in this area is surprisingly limited. The primary research focus has been on the more obvious cognitive and neuropsychological changes immediately following brain trauma. A recent study of military personnel with a past history of mild traumatic brain injury (mTBI) identified a significant relationship between visual complaints and concussion, even five years post-injury. ${ }^{13}$ This suggests that clinicians must remain aware of possible visual dysfunction years after the event, particularly in more traumatic cases. The incidence of visual symptoms appears to remain relatively consistent across mTBI of different causes and intensities. ${ }^{14}$

\section{Accommodation}

Accommodation refers to a shift in the focus of the eye to maintain a clear image of an object as it varies its distance from the observer, primarily as it approaches. The neural control of accommodation requires afferent input to the visual cortex, and brainstem control of eye movements and the ciliary muscle. The complexity of this pathway makes it potentially susceptible to damage, with resultant dysfunction after a concussion. Within the clinical setting, this may manifest as complaints of blurred vision, asthenopia (tired eyes) and difficulty reading. ${ }^{15}$

Master et al showed reduced accommodative amplitudes in $51 \%$ of young patients with concussion when compared with age-matched normative values. ${ }^{11}$ Similarly, Capo-Aponte et al prospectively examined 20 military personnel following $\mathrm{mTBI}$ and reported

\section{Table 1. Symptoms and signs affecting the visual system following a concussion}

\begin{tabular}{ll}
\hline Symptoms & Signs \\
\hline - Loss of vision & - Reduced distance or reading acuity \\
- Blurred vision & - Anisocoria \\
- Double vision & - Impaired vestibulo-ocular reflex \\
- Reading difficulties & - Slowed or reduced eye movements \\
- Photophobia & - Impaired convergence \\
- Headaches with visual tasks & - Impaired accommodation \\
- Difficulty tracking fast objects & - Decreased visual attention \\
\hline
\end{tabular}

reduced amplitudes of accommodation and accommodative facility in $65 \%$ and $35 \%$ of patients, respectively. ${ }^{12}$

Accommodative capacity is age dependent. Healthy young or adolescent patients can maintain clear focus on near objects (as close as $<10 \mathrm{~cm}$ ); however, this capacity progressively reduces with age as the crystalline lens gradually becomes more rigid. Therefore, younger patients may be more affected by accommodation dysfunction of neurological origin than older patients. The level of accommodation can also vary with refractive error such as myopia, in which accommodative lag is commonly reported; therefore, a history of wearing glasses or using contact lenses is important adjunctive information.

Accommodation is tested by slowly bringing text towards the subject until the point of blur. This distance is then matched against age-related norms. Specialist equipment (Royal Air Force [RAF] Rule) is designed to measure accommodative amplitudes within ophthalmic practices. However, in the general practice setting, a finding of gross accommodation or a significant reduction through repeated measures in conjunction with symptoms including difficulty concentrating, headaches following a relatively short period of near work or the appearance of jumbled words when reading - would necessitate referral to a neuro-ophthalmologist for further measurement and possible rehabilitation.

\section{Convergence}

Convergence is the simultaneous movement of both eyes inwards to maintain binocular fixation on a target (Figure 1). Cortical areas including the visual cortex, parietal lobe, frontal eye fields, supra-oculomotor area and cerebellum are involved in the convergence pathway. ${ }^{16}$ This neurological pathway works closely with accommodation to provide concurrent clear focus on near objects and is highly susceptible to damage by concussion. Convergence abnormalities are reported in $14-55 \%$ of athletes, ${ }^{17-19} 48-55 \%$ of military personnel ${ }^{11,20}$ and $14-49 \%$ of 
outpatient groups ${ }^{11,21,22}$ previously diagnosed with concussion. Poor convergence will manifest clinically with double vision following near work, and blurred vision or asthenopia with prolonged near tasks such as reading.

Up to $40 \%$ of patients have ongoing visual symptoms suggestive of convergence dysfunction for at least one month following the concussive episode. ${ }^{23}$ This suggests high visual morbidity, potentially causing significant disruption to reading and computer work. For athletes, this may affect performance such as their ability to track a ball. For military or security personnel, these symptoms could be particularly dangerous when undertaking rapid-response activities.

Convergence is tested by slowly bringing a pen (or target) closer to the patient's eyes (Figure 1). The patient is asked to maintain a single image and warned that the target may become blurred as it is brought forward (because of accommodative ability). Clinicians should look for a break in binocular fusion, which would manifest with an obvious divergence of one or both eyes from the target and/or the patient reporting diplopia. It is important to note that approximately $0.2 \%$ of the general population will have an abnormal convergence near point, clinically referred to as convergence insufficiency. ${ }^{24} \mathrm{~A}$ diagnosis of clinicallyreduced convergence may indicate a range of interconnected conditions of the oculomotor system..$^{25}$ Therefore, referral to an ophthalmologist or orthoptist is recommended.

\section{Saccades}

Saccades are rapid eye movements between two or more separate fixation points, at any angular position. Types of saccades are listed in Table 2 . The
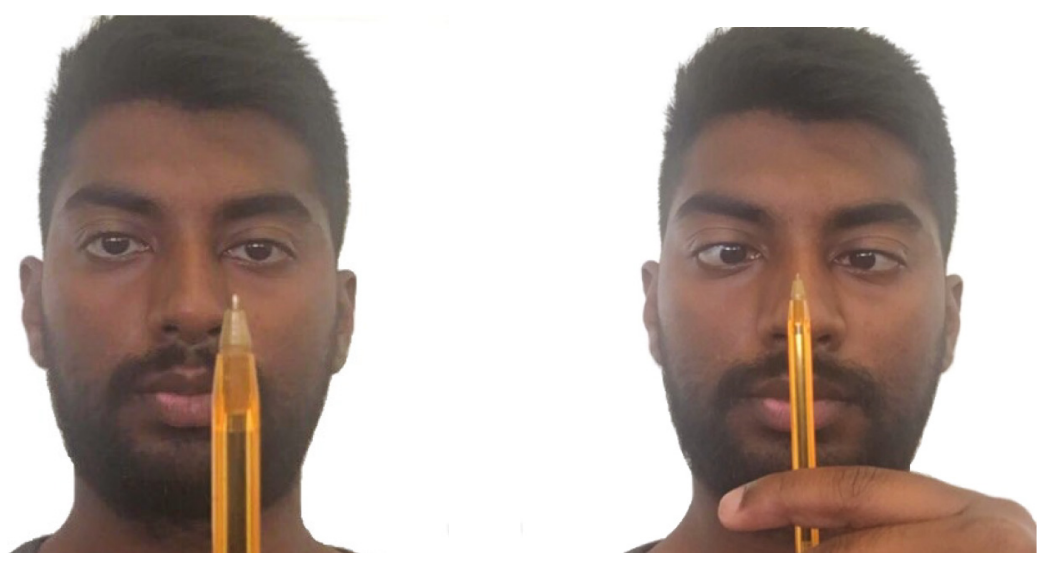

Figure 1. Eyes before (left) and after (right) converging on a pen. Convergence is tested by measuring the convergence near point.

\section{Table 2. Types of saccades}

\begin{tabular}{ll}
\hline Type of saccade & Definition \\
\hline Visually guided saccade & Eyes move toward a visual stimulus \\
\hline Anti-saccade & Eyes move away from visual stimulus \\
\hline Memory-guided saccade & Eyes move toward a remembered location \\
\hline Predictive saccade & Eyes are kept on a target moving in a predictive spatial manner \\
\hline
\end{tabular}


attention faculties. ${ }^{30}$ The test can be compared with a baseline finding if available, or to age-appropriate normative values. The King-Devick test has been increasingly used within general practice offices, particularly those with sporting associations, for diagnostic purposes and to track recovery as part of a return-toplay protocol.

\section{Smooth pursuit eye movements}

Smooth pursuit eye movements are performed to closely track a moving target, holding the image of the moving target steady on the fovea. As with other types of ocular movement, smooth pursuit requires the integration of multiple cortical areas and is similarly susceptible to concussion.

Few papers discuss concussion-related smooth pursuit dysfunction. However, those that exist report high rates of abnormalities. ${ }^{12,20,31}$ Within the clinical setting, this would result in increased difficulty following moving objects, such as when catching a ball and tracking nearby cars while driving. Examining smooth pursuits can be achieved by asking the patient to track a pen across the vertical and horizontal planes. Jerky movements or failure to adequately follow

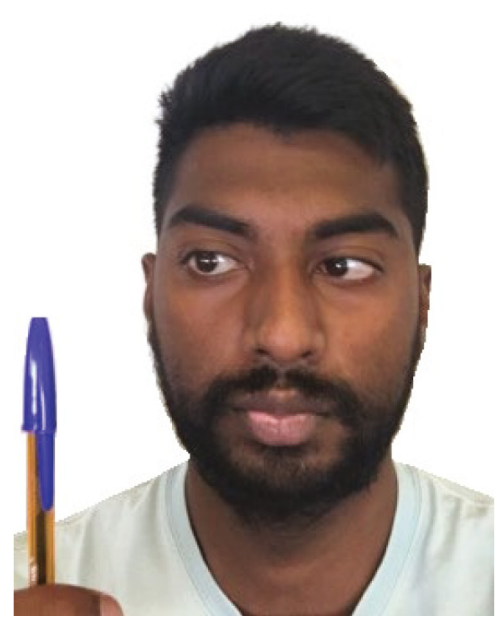

Figure 2. For horizontal saccade testing, patients fixate continuously between a target on their right and left. The examiner observes for the complete and simultaneous movement of both eyes to each target. The same can be applied for vertical saccade testing, with a pen held high and low.

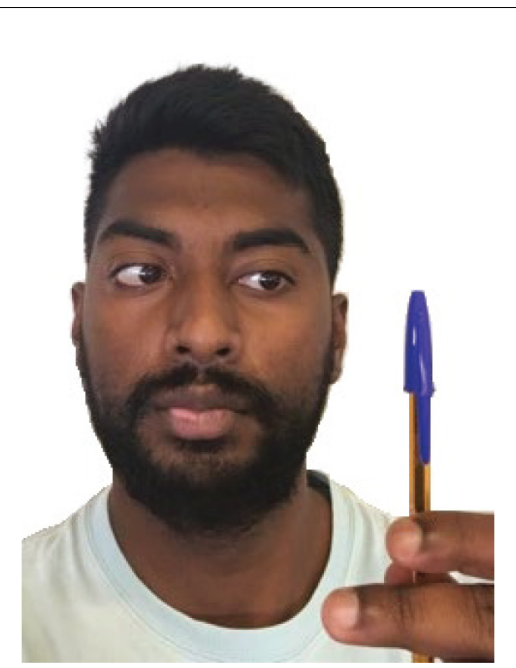

the target may indicate problems with extensive ophthalmological training, assessing subtle defects in smooth pursuit eye movements can be challenging in clinical practice. Specialist referral is suggested if smooth pursuit or tracking dysfunction is suspected.

In the research setting, using video-oculography to track disconjugate eye movements is gaining popularity as a possible marker for concussion. ${ }^{32}$ Maruta et al examined a population with chronic concussive symptoms and found a significant decline in their predictive visual tracking capabilities. ${ }^{33,34}$ Using diffusion tensor imaging, researchers attributed these oculomotor impairments to white matter tract damage in the corona radiata, left superior cerebellar peduncle and genu of the corpus collosum. These patients also showed reduced attention and working memory, highlighting the potential need for multidisciplinary referral to both ophthalmologists and neurologists for more extensive examination. A greater understanding of video-oculography metrics and the ability to provide a portable, readily accessible model will help facilitate translation of this technology into the clinic. ${ }^{35}$ smooth pursuit movements. Even with (1)

\section{Other conditions}

Visual field anomalies have been reported following significant trauma but are rare following sports-related concussive episodes. ${ }^{14,35}$ Visual field defects related to significant trauma include homonymous hemianopia and quadrantanopia. A confrontation field test can be performed in the clinic if field loss is suspected, with referral for formal visual field testing if there are any concerns.

Cranial nerve palsy may also occur following severe trauma. ${ }^{14}$ The patient would present with a manifest strabismus, and report diplopia.

Visual field loss and cranial neuropathy are not expected in concussion and should prompt further neurological examination and neuroimaging for a structural pathology.

\section{Oculomotor rehabilitation}

Although research is quite limited, there is some evidence supporting vision therapy/exercises as an effective strategy for rehabilitation. ${ }^{36,21}$ Current protocols in Australian sport recommend rest and a step-wise return-to-play guideline. ${ }^{37}$ However, recovery will be dependent on many factors including the number and severity of the existing ocular defects. Ciuffreda et al found that treatment in the form of eye tracking, accommodation and convergence exercises may expedite recovery and completely alleviate symptoms in almost $90 \%$ of patients with mTBI who have persistent oculomotor defects. ${ }^{36}$ This can require between 10 and 30 vision therapy sessions over an eight-month period until symptoms resolve. ${ }^{36}$

\section{Conclusion}

Many forms of ocular dysfunction are found in acute and chronic cases of concussion. Being the primary medical contact for many patients with a concussion, either in the immediate or short-term period following the initial trauma, GPs need to be vigilant about the ocular dysfunction, as well as the behavioural, physical and emotional 
symptoms associated with concussion. Practical tips for assessment of ocular dysfunction following concussion are provided in Box 1. If the GP suspects visual and oculomotor impairment, referral to an ophthalmologist will provide patients with a complete ocular assessment and earlier implementation of treatment and rehabilitation.

\section{Box 1. Practical tips for concussion assessments}

- Subtle oculomotor dysfunction may be described by the patient as:

- increased difficulty when completing near tasks

- headache and eye strain after shorter duration of visual effort.

- Standard assessment of extra-ocular muscles and pupillary response can be supplemented with tests for:

- convergence

- accommodation

- saccadic eye movement and smooth pursuits.

- Signs and symptoms can persist in some patients for weeks or months after concussion.

- Visual field loss and cranial nerve palsies are rare in concussion and should be investigated further.

\section{Key points}

- GPs must be alert to persistent oculomotor dysfunction following concussion including impairments to accommodation, convergence, smooth pursuits and saccadic eye movements.

- Developing strong relationships between GPs and ophthalmologists is important to provide the best evidence-based care for patients with concussion-related visual dysfunction.

- Patients with persistent visual disturbances may benefit from oculomotor rehabilitation, such as convergence, accommodation and eye tracking exercises.

\section{Authors}

Premkumar Gunasekaran BMedSc, MOrth, HDR candidate, Discipline of Orthoptics, Graduate School of Health, University of Technology Sydney, NSW
Christopher Hodge BAppSc, PhD, Honorary Associate, Discipline of Orthoptics, Graduate School of Health, University of Technology Sydney, NSW Clinical Senior Lecturer, University of Sydney, Save Sight Institute, Discipline of Ophthalmology, Sydney Medical School, NSW

Kathryn Rose DOBA, DipAppSci (Orth), GradDipNeurosci, PhD, Head of Discipline, Orthoptics, Discipline of Orthoptics, Graduate School of Health, University of Technology Sydney, NSW

Clare L Fraser MBBS, MMed, FRANZCO, Associate Professor in Neuro-ophthalmology, University of Sydney, Save Sight Institute, Discipline of Ophthalmology, Sydney Medical School, NSW. clare.fraser@sydney.edu.au

Competing interests: None.

Funding: None.

Provenance and peer review: Commissioned, externally peer reviewed.

\section{References}

1. Helps Y, Henley G, Harrison JE. Hospital separations due to traumatic brain injury, Australia 2004-05. Injury research and statistics series number 45 (Cat no. INJCAT 116). Adelaide: AlHW, 2018. Available at www.aihw.gov.au/getmedia/61decf32-937b4280-8571-dd66061c99bf/hsdttbia04-05.pdf. aspx?inline=true [Accessed 21 June 2019].

2. Finch CF, Clapperton AJ, McCrory P. Increasing incidence of hospitalisation for sport-related concussion in Victoria, Australia. Med J Aust 2013;198(8):427-30.

3. McCrory P, Meeuwisse W, Dvořák J, et al. Consensus statement on concussion in sport the 5th international conference on concussion in sport held in Berlin, October 2016. Br J Sports Med 2017;51(11):838-47. doi: 10.1136/ bjsports-2017-097699.

4. Simpson-Jones ME, Hunt AW. Vision rehabilitation interventions following mild traumatic brain injury: a scoping review. Disabil Rehabil 2019;41(18):2206-22 doi: 10.1080/09638288.2018.1460407.

5. Meehan WP 3rd, d'Hemecourt P, Collins CL, Comstock RD. Assessment and management of sport-related concussions in United States high schools. Am J Sports Med 2011;39(11):2304-10. doi: 10.1177/0363546511423503.

6. Halstead ME, Walter KD; Council on Sports Medicine and Fitness. American Academy of Pediatrics. Clinical report - Sport-related concussion in children and adolescents. Pediatrics 2010;126(3):597-615. doi: 10.1542/peds.2010-2005.

7. Bin Zahid A, Hubbard ME, Dammavalam VM, et al. Assessment of acute head injury in an emergency department population using sport concussion assessment tool - 3rd edition. Appl Neuropsychol Adult;25(2):110-19. doi: 10.1080/23279095.2016.1248765.

8. Elkington L, Manzanero S, Hughes D. Concussion in sport australia position statement. Bruce, ACT: Australian Government, 2019. Available at www.sportaus.gov.au/_data/assets/ pdf file/0005/683501/February 2019 Concussion_Position_Statement_AC.pdf [Accessed 27 June 2019]

9. Delaney JS, Abuzeyad F, Correa JA, Foxford R Recognition and characteristics of concussions in the emergency department population. J Emerg Med 2005;29(2):189-97.

10. Debacker J, Ventura R, Galetta SL, Balcer LJ, Rucker JC. Neuro-ophthalmologic disorders following concussion. Handb Clin Neurol 2018;158:145-52. doi: 10.1016/B978-0-444-639547.00015-X.
11. Master CL, Scheiman M, Gallaway M, et al. Vision diagnoses are common after concussion in adolescents. Clin Pediatr (Phila) 2016:55(3):260-67. doi: 10.1177/0009922815594367.

12. Capo-Aponte JE, Urosevich TG, Temme LA, Tarbett AK, Sanghera NK. Visual dysfunctions and symptoms during the subacute stage of blast-induced mild traumatic brain injury. Mil Med 2012;177(7):804-13.

13. Urosevich TG, Boscarino JJ, Hoffman SN, et al. Visual dysfunction and associated co-morbidities as predictors of mild traumatic brain injury seen among veterans in non-VA facilities: Implications for clinical practice. Mil Med 2018;183(11-12):e564-70. doi: 10.1093/milmed/usy102.

14. Armstrong RA. Visual problems associated with traumatic brain injury. Clin Exp Optom 2018;101(6):716-26. doi: 10.1111/cxo.12670.

15. Daum K. Accommodative dysfunction. Doc Ophthalmol 1983;55(3):177-98.

16. Rowe FJ. Clinical orthoptics, 3rd edn. Chichester UK: John Wiley \& Sons, 2012.

17. Howell DR, O Brien MJ, Raghuram A, Shah AS, Meehan WP 3rd. Near point of convergence and gait deficits in adolescents after sport-related concussion. Clin J Sport Med 2018;28(3):262-67. doi: 10.1097/JSM.0000000000000439.

18. Vernau BT, Grady MF, Goodman A, et al. Oculomotor and neurocognitive assessment of youth ice hockey players: baseline associations and observations after concussion. Dev Neuropsychol 2015;40(1):7-11. doi: 10.1080/87565641.2014.971955.

19. Pearce KL, Sufrinko A, Lau BC, Henry L, Collins MW, Kontos AP. Near point of convergence after a sport-related concussion: Measurement reliability and relationship to neurocognitive impairment and symptoms. Am J Sports Med 2015;43(12):3055-61. doi: 10.1177/0363546515606430.

20. Brahm KD, Wilgenburg HM, Kirby J, Ingalla S, Chang CY, Goodrich GL. Visual impairment and dysfunction in combat-injured servicemembers with traumatic brain injury. Optom Vis Sci 2009;86(7):817-25. doi: 10.1097/ OPX.0b013e3181adff2d.

21. Gallaway M, Scheiman M, Mitchell GL. Vision therapy for post-concussion vision disorders. Optom Vis Sci 2017:94(1):68-73. doi: 10.1097/ OPX.0000000000000935

22. Storey EP, Master SR, Lockyer JE, Podolak OE Grady MF, Master CL. Near point of convergence after concussion in children. Optom Vis Sci 2017;94(1):96-100. doi: 10.1097/ OPX.0000000000000910.

23. Mittenberg W, Canyock EM, Condit D, Patton C. Treatment of post-concussion syndrome following mild head injury. J Clin Exp Neuropsychol 2001;23(6):829-36.

24. Arnoldi K, Reynolds JD. A review of convergence insufficiency: What are we really accomplishing with exercises? Am Orthopt J 2007;57:123-30. doi: 10.3368/aoj.57.1.123.

25. Raghuram A, Cotter S, Gowrisankaran S, et al. Post-concussion: receded near point of convergence is not diagnostic of convergence insufficiency. Am J Ophthalmol 2019; pii: S00029394(19)30182-5. doi: 10.1016/j.ajo.2019.04.008.

26. Diwakar M, Harrington DL, Maruta J, et al. Filling in the gaps: Anticipatory control of eye movements in chronic mild traumatic brain injury. Neuroimage Clin 2015;8:210-23. doi: 10.1016/j.nicl.2015.04.011

27. Rizzo JR, Hudson TE, Dai W, et al. Rapid number naming in chronic concussion: Eye movements in the King-Devick test. Ann Clin Transl Neurol 2016;3(10):801-11. 
28. Heitger $\mathrm{MH}$, Anderson TJ, Jones RD,

Dalrymple-Alford JC, Frampton CM, Ardagh MW. Eye movement and visuomotor arm movement deficits following mild closed head injury. Brain 2004;127(Pt 3):575-90.

29. Heitger MH, Jones RD, Macleod AD, Snell DL, Frampton CM, Anderson TJ. Impaired eye movements in post-concussion syndrome indicate suboptimal brain function beyond the influence of depression, malingering or intellectual ability. Brain 2009;132(Pt 10):2850-70.

30. Galetta KM, Liu M, Leong DF, Ventura RE, Galetta SL, Balcer LJ. The King-Devick test of rapid number naming for concussion detection: Meta-analysis and systematic review of the literature. Concussion 2015;1(2):CNC8. doi: 10.2217/cnc.15.8.

31. Cohen D, Conidi F. Neurologic exam findings and clinical manifestations of post-concussion syndrome in a pediatric population (P7.117). Neurology Apr 2015;84(Suppl 14).

32. Snegireva N, Derman W, Patricios J, Welman KE. Eye tracking technology in sports-related concussion: A systematic review and metaanalysis. Physiol Meas 2018;39(12):12TR01. doi: 10.1088/1361-6579/aaef44.

33. Maruta J, Suh M, Niogi SN, Mukherjee $P$, Ghajar J. Visual tracking synchronization as a metric for concussion screening. J Head Trauma Rehabil 2010;25(4):293-305. doi: 10.1097/ HTR.0b013e3181e67936.

34. Maruta J, Ghajar J. Detecting eye movement abnormalities from concussion. Prog Neurol Surg 2014;28:226-33. doi: 10.1159/000358786.

35. Jacobs SM, Van Stavern GP. Neuro-ophthalmic deficits after head trauma. Curr Neurol Neurosci Rep 2013;13(11):389. doi: 10.1007/s11910-0130389-5.

36. Ciuffreda KJ, Rutner D, Kapoor N, Suchoff IB, Craig S, Han ME. Vision therapy for oculomotor dysfunctions in acquired brain injury: $A$ retrospective analysis. Optometry 2008;79(1):18-22.

37. Gunasekaran $P$, Hodge $C$, Pearce A, King D, Fraser $C$. A review of concussion diagnosis and management in Australian professional sporting codes. Phys Sportsmed 2019:1-7. doi: 10.1080/00913847.2019.1624656. 